\title{
PROJECTIONS ON HARDY SPACES \\ IN THE LIE BALL
}

\author{
DAVID BEKOLLÉ
}

\begin{abstract}
On the Lie ball $\omega$ of $\mathbb{C}^{n}, n \geq 3$, we prove that for all $p \in[1, \infty)$, $p \neq 2$, the Hardy space $H^{p}(\omega)$ is an uncomplemented subspace of the Lebesgue space $L^{p}\left(\partial_{0} \omega, d \sigma\right)$, where $\partial_{0} \omega$ denotes the Shilov boundary of $\omega$ and $d \sigma$ is a normalized invariant measure on $\partial_{0} \omega$.
\end{abstract}

\section{Introduction}

Let $\omega=\omega_{n}$ denote the Lie ball of $\mathbb{C}^{n}, n \geq 3$, defined by

$$
\omega=\left\{z \in \mathbb{C}^{n}: 1-2|z|^{2}+\left|\sum_{j=1}^{n} z_{j}^{2}\right|^{2}>0 \text { and }\left|\sum_{j=1}^{n} z_{j}^{2}\right|<1\right\} .
$$

The Shilov boundary $\partial_{0} \omega$ of $\omega$ is $\partial_{0} \omega=\left\{e^{i \theta} x: \theta \in[0,2 \pi)\right.$ and $\left.x \in S_{n-1}\right\}$, where $S_{n-1}$ denotes the unit sphere of $\mathbb{R}^{n}$. Denote by $G$ the compact group consisting of the following transformations in $\mathbb{C}^{n}: g$ belongs to $G$ if there are $h \in S O(n)$ and $\theta \in[0,2 \pi)$ such that $g(z)=e^{i \theta} h(z)$ for all $z \in \mathbb{C}^{n}$. The group $G$ is subgroup of the automorphism group of $\omega$ and $G$ acts transitively on $\partial_{0} \omega$. Moreover, a normalized $G$-invariant measure on $\partial_{0} \omega$ is $d \sigma\left(e^{i \theta} x\right)=\frac{\Gamma(n / 2)}{4 \pi^{n / 2+1}} d \theta d \mu(x)$, where $d \mu$ denotes the Lebesgue surface measure on $S_{n-1}$.

Recall that the Hardy space $H^{p}(\omega), 1 \leq p<\infty$, is defined as consisting of all functions $f$ holomorphic in $\omega$ and such that

$$
\sup _{0<r<1} \int_{\partial_{0} \omega}|f(r z)|^{p} d \sigma(z)<\infty .
$$

Every $f \in H^{p}(\omega)$ has radial limits $\sigma$-a.e. on $\partial_{0} \omega$ and when we identify $f$ with its boundary value function, the space $H^{p}(\omega)$ can be viewed as a closed subspace of $L^{p}\left(\partial_{0} \omega, d \sigma\right)$. For this fact, c.f. e.g. [V].

The main result of the present paper is the following: 
Theorem 1.1. For all $p \in[1, \infty), p \neq 2, H^{p}(\omega)$ is an uncomplemented subspace of $L^{p}\left(\partial_{0} \omega, d \sigma\right)$.

In Chapter 12 of [R1], the same result is proved for $p=1$ in the unit ball of $\mathbb{C}^{n}$. It turns out that the result for the Lie ball (Theorem 1.1) can be deduced exactly in the same way from the following theorem:

Theorem 1.2 [BeBo]. In the Lie ball $\omega$ of $\mathbb{C}^{n}, n \geq 3$, the Szegö projection is $L^{p}$-unbounded for all $p \in(1, \infty) p \neq 2$.

We recall that the Szegö projection for $\omega$ is the orthogonal projection of $L^{2}\left(\partial_{0} \omega, d \sigma\right)$ onto $H^{2}(\omega)$.

Let $U$ denote the unit disc in the complex plane. Then the following question is open: are the Hardy spaces $H^{p}(\omega)$ and $H^{p}(U)$ isomorphic for $p \in(1, \infty), p \neq 2$ ? P. Wojtaszczyk [W] answered this question affirmatively in the unit ball $B_{n}$ of $\mathbb{C}^{n}, n \geq 2$. His proof uses the fact that $H^{p}\left(B_{n}\right)$ is a complemented subspace of $L^{p}\left(\partial B_{n}\right)$, by a theorem of $[\mathbf{K V}]$, and hence it cannot be applied to the Lie ball case. On the other hand, T. Wolniewicz [Wo] proved that for classical domains $D$ of class I (in Hua's standard notation $[\mathbf{H}]$ ), the Hardy space $H^{1}(D)$ is not isomorphic to $H^{1}(U)$ except when $D$ is a unit ball. In particular, $H^{1}(D)$ is not isomorphic to $H^{1}(U)$ when $D$ is the Lie ball $\omega_{4}$ in $C^{4}$. It seems unlikely that $H^{1}\left(\omega_{n}\right)$ is isomorphic to $H^{1}(U)$ when $n=3$ and $n \geq 5$ (i.e. for domains of class IV).

Finally, it seems to be certain that Theorems 1.1 and 1.2 can be extended to all bounded symmetric domains of tube type. To do this, an appropriate reference would be $[\mathbf{K}]$.

In the second section, we recall a decomposition of $L^{2}\left(\partial_{0} \omega, d \sigma\right)$ into a direct sum of $G$-invariant subspaces (c.f. e.g. [BCK]). We denote by $N$ the polynomial on $\mathbb{C}^{n}$ defined by

$$
N(z)=\sum_{j=1}^{n} z_{j}^{2}
$$

and by $\square$ the differential polynomial defined by

$$
\square_{z}=\sum_{j=1}^{n} \frac{\partial^{2}}{\partial z_{j}^{2}} .
$$

Denote by $H_{k}, k \in \mathbb{Z}_{+}$, the space consisting of restrictions to $\partial_{0} \omega$ of homogeneous holomorphic polynomials $p$ of degree $k$ such that $\square p=0$. 
Then $L^{2}\left(\partial_{0} \omega, d \sigma\right)=\sum_{(j, k) \in \mathbb{Z} \times \mathbb{Z}_{+}} \oplus N^{j} H_{k}$ and the spaces $N^{j} H_{k}$ are pairwise orthogonal with respect to the usual inner product in $L^{2}\left(\partial_{0} \omega, d \sigma\right)$.

In the third section, we study the orthogonal projection of $L^{2}\left(\partial_{0} \omega, d \sigma\right)$ into $N^{j} H_{k}$ and linear maps $T: N^{j_{1}} H_{k_{1}} \rightarrow N^{j_{2}} H_{k_{2}}$ that commute with $G$.

In the fourth section, we characterize the $G$-invariant closed subspaces of $L^{2}\left(\partial_{0} \omega, d \sigma\right), 1 \leq p<\infty$ and of $\mathcal{C}\left(\partial_{0}, \omega\right)$. This extends a theorem due to A. Nagel and W. Rudin (Theorem 12.3.6 in [R1]) for the unit ball $B_{n}$ of $\mathbb{C}^{n}$. Denote by $H(p, q),(p, q) \in \mathbb{Z}_{+} \times \mathbb{Z}_{+}$the space of homogeneous harmonic polynomials on $\mathbb{C}^{n}$ that have total degree $p$ in the variables $z_{1}, \ldots, z_{n}$, and total degree $q$ in the variables $\bar{z}_{1}, \ldots, \bar{z}_{n}$. Then $L^{2}\left(\partial B_{n}\right)$ is the direct sum of the spaces $H(p, q)$ and the theorem of Nagel and Rudin in $B_{n}$ is stated in terms of the $H(p, q)$ spaces. Their proof still works in the Lie ball case, when the spaces $H(p, q)$ are replaced by the spaces $N^{j} H_{k}$.

Finally, in the fifth section, we prove Theorem 1.1.

\section{Decomposition of $L^{2}\left(\partial_{0} \omega, d \sigma\right)$}

When we identify $\mathbb{C}^{n}$ with $\mathbb{R}^{2 n}$, we let $\mathcal{P}$ denote the space of all complex-valued polynomials on $\mathbb{C}^{n}$. By the Stone-Weierstrass Theorem, the space of restrictions to $\partial_{0} \omega$ of elements of $\mathcal{P}$ is dense in $\mathcal{C}\left(\partial_{0} \omega\right)$, and hence in $L^{2}\left(\partial_{0} \omega, d \sigma\right)$. Denote by $\mathcal{P}^{(k)}$ the space of restrictions to $\partial_{0} \omega$ of homogeneous complex-valued polynomials of degree $k$; then

$$
L^{2}\left(\partial_{0} \omega, d \sigma\right)=\sum_{k=0}^{+\infty} \mathcal{P}^{(k)}
$$

Let $N$ be the polynomial on $\mathbb{C}^{n}$ defined by (1); then

$$
\left.\bar{z}_{j}\right|_{\partial_{0} \omega}=\left.N(z)^{-1} z_{j}\right|_{\partial_{0} \omega} .
$$

Furthermore, when $P_{k}$ denote the space of restrictions to $\partial_{0} \omega$ of homogeneous holomorphic polynomials of degree $k$ on $\mathbb{C}^{n}$, we obtain:

$$
\mathcal{P}^{(k)}=\sum_{j=0}^{k} \oplus N^{-j} P_{k} .
$$

For $q \in P_{k}, q(z)=\sum_{\alpha \in A} b_{\alpha} z^{\alpha}$, define $\tilde{q}$ by $\tilde{q}(z)=\sum_{\alpha \in A} \tilde{b}_{\alpha} z^{\alpha}$ and equip $P_{k}$ with the differential inner product

$$
(p \mid q)=\frac{1}{k !} p(D) \tilde{q},
$$


where $p(D)=\sum_{\alpha \in A} a_{\alpha}\left(\frac{\partial}{\partial z_{1}}\right)^{\alpha_{1}} \ldots\left(\frac{\partial}{\partial z_{n}}\right)^{\alpha_{n}}$ and $A=\left\{\alpha=\left(\alpha_{1}, \ldots, \alpha_{n}\right)\right.$ : $|\alpha|=k\}$.

Denote by $H_{k}, k \geq 2$, the orthogonal of $N P_{k-2}$ in $P_{k}$ relative to the inner product (|) and set $H_{1}=P_{1}, H_{0}=P_{0}$.

We have the following lemma:

Lemma 2.1. The space $H_{k}$ consists of all restrictions to $\partial_{0} \omega$ of homogeneous holomorphic polynomials of degree $k$ that are annihilated by the differential polynomial $\square$ defined in (2).

Moreover,

$$
P_{k}=\sum_{j=0}^{[k / 2]} \oplus N^{j} H_{k-2 j}
$$

Proof: This is just Lemma 3.2 in [Co We].

In the sequel, the letter $Q$ will stand for $\mathbb{Z} \times \mathbb{Z}_{+}$. We prove next the following lemma:

Lemma 2.2. For all $(j, k) \in Q$, the space $N^{j} H_{k}$ is $G$-invariant.

Proof: We must prove that $\left(N^{j} p\right) \circ \phi \in N^{j} H_{k}$ for all $\phi \in G$ and $p \in H_{k}$. There is a constant $c_{j}(\phi)$ such that $\left(N_{j} p\right) \circ \phi=c_{j}(\phi) N^{j} .(p \circ \phi)$, and hence it suffices to prove that $p \circ \phi \in H_{k}$. But $\square$ commutes with $G$, i.e. $\square(p \circ \phi)=(\square p) \circ \phi$ for all $\phi \in G$ and $p \in P_{k}$. Then, $\square p=0$ implies $\square(p \circ \phi)=0$ and the desired conclusion follows from the first assertion of Lemma 2.1.

In the rest of the paper, $L^{2}\left(\partial_{0} \omega, d \sigma\right)$ will be equipped with the usual inner product:

$$
[f, g]=\int_{\partial_{0} \omega} f \bar{g} d \sigma .
$$

We prove the following proposition:

Proposition 2.3. The spaces $N^{j} H_{k},(j, k) \in Q$, are pairwise orthogonal with respect to the inner product [ , ].

Proof: For all $\left(j_{1}, k_{1}\right)$ and $\left(j_{2}, k_{2}\right)$ in $Q$ such that $\left(j_{1}, k_{1}\right) \neq\left(j_{2}, k_{2}\right)$ and for all $p \in H_{k_{1}}$ and $q \in H_{k_{2}}$, we have:

$$
\left[N^{j_{1}} p, N^{j_{2}} q\right]=\left(\int_{0}^{2 \pi} e^{2 i \theta\left(j_{1}+k_{1}-j_{2}-k_{2}\right)} d \theta\right)\left(\int_{S_{n-1}} p \bar{q} d \mu\right) .
$$


Assume first that $k_{1} \neq k_{2}$. On $\mathbb{R}^{n}, p$ and $q$ are homogeneous harmonic polynomials of respective degrees $k_{1}$ and $k_{2}$; it is known (cf. e.g. [R1, p. 254]) that the integral over $S_{n-1}$ is then zero. Assume next that $k_{1}=k_{2}$ and $j_{1} \neq j_{2}$. In this case, the integral over $[0,2 \pi)$ is zero. This proves the proposition.

We deduce next the following corollary:

Corollary 2.4. The space $L^{2}\left(\partial_{0} \omega, d \sigma\right)$ is the direct sum of the pairwise orthogonal subspaces $N^{j} H_{k},(j, k) \in Q$.

Proof: In view of Proposition 2.3, it is enough to prove that $L^{2}\left(\partial_{0} \omega, d \sigma\right)$ is contained in the sum of the spaces $N^{j} H_{k},(j, k) \in Q$. Combining (4) and (6) yields:

$$
\mathcal{P}^{(k)}=\sum_{\substack{j=0,1, \ldots, k \\ i=0,1, \ldots,\left[\frac{k}{2}\right]}} \oplus N^{-j+i} H_{k-2 i} .
$$

The conclusion then follows from (3). This completes the proof.

\section{The spaces $N^{j} H_{k}$}

Denote by $S$ the representation of $G$ in $\mathcal{P}$ defined by $S_{\phi} p(z)=p\left(\phi^{-1}(z)\right)$ and denote by $S_{j k}$ the restriction of $S$ to $N^{j} H_{k}$. We have the following theorem:

Theorem 3.1. The representation $S_{j k}$ of $G$ in $N^{j} H_{k}$ is irreducible, i.e. $N^{j} H_{k}$ has no proper $G$-invariant subspace.

Proof: Since $S O(n) \subset G$, it is known (cf. Theorem 3.3 in [Co We]) that the representation $S_{o k}$ in $H_{k}$ is irreducible. On the other hand, $N^{-j} Y$ is a $G$-invariant subspace of $H_{k}$ whenever $Y$ is a $G$-invariant subspace of $N^{j} H_{k}$. The conclusion follows.

Fix $(j, k) \in Q$ and denote by $\pi_{j k}$ the orthogonal projection of $L^{2}\left(\partial_{0} \omega, d \sigma\right)$ onto its closed (since it is finite-dimensional) subspace $N^{j} H_{k}$. We prove first the following lemma:

Lemma 3.2. The projection $\pi_{j k}$ commutes with $G$, i.e. for all $\phi \in G$ and $f \in L^{2}\left(\partial_{0} \omega, d \sigma\right), \pi_{j k}(f \circ \phi)=\left(\pi_{j k} f\right) \circ \phi$.

Proof: This is an immediate consequence of the $G$-invariance of $N^{j} H_{k}$ (Lemma 2.2) and $\left(N^{j} H_{k}\right)^{\perp}$.

We prove next the following theorem: 
Theorem 3.3. 1. To every $z \in \partial_{0} \omega$ corresponds a unique $K_{z} \in N^{j} H_{k}$ that satisfies

$$
\left(\pi_{j k} f\right)(z)=\left[f, K_{z}\right] \quad\left(f \in L^{2}\left(\partial_{0} \omega, d \sigma\right)\right) .
$$

2. These kernel functions $K_{z}$ have the following addtional properties:

$$
\begin{array}{rr}
K_{z}(w)=\overline{K_{w}(z)} & \left(z, w \in \partial_{0} \omega\right), \\
\pi_{j k} f=\int_{\partial_{0} \omega} f(\zeta) K_{\zeta} d \sigma(\zeta) & \left(f \in L^{2}\left(\partial_{0} \omega, d \sigma\right)\right. \\
K_{\phi(z)}=K_{z} \circ \phi^{-1} & (\phi \in G),
\end{array}
$$

for all $\Psi \in G$ that fix $z$, and

$$
K_{z}(z)=K_{w}(w)>0 \quad\left(z, w \in \partial_{0} \omega\right) .
$$

Proof: We write $\pi$ for $\pi_{j k}$.

1. In the finite-dimensional space $N^{j} H_{k}$, the norms $\max _{\partial_{0} \omega}\left|N^{j} p\right|$ and $\left\|N^{j} p\right\|$ are equivalent.

The correspondence $f \rightarrow(\pi f)(z)$ is then a bounded linear functional on $L^{2}\left(\partial_{0} \omega, d \sigma\right)$. Thus there is a unique $K_{z} \in L^{2}\left(\partial_{0} \omega, d \sigma\right)$ that satisfies (8). Since $\pi f=0$ for every $f \in\left(N^{j} H_{k}\right)^{\perp}$, it follows that $K_{z} \in N^{j} H_{k}$.

2. For $f=K_{w},(8)$ becomes

$$
K_{w}(z)=\left[K_{w}, K_{z}\right]=\left[\overline{K_{z}, K_{w}}\right]=\overline{K_{z}(w)} .
$$

This proves (9) and (9) shows that (10) is just another way of writing (8).

Since $\pi$ commutes with $G$ (Lemma 3.2), $(\pi f)(\phi(z))=\pi(f \circ \phi)(z)=$ $\left[f \circ \phi, K_{z}\right]=\left[f, K_{z} \circ \phi^{-1}\right]$ for every $f \in L^{2}\left(\partial_{0} \omega, d \sigma\right)$. Combined with (8), this proves (11), hence also its special case (12).

Finally,

$$
\left.K_{\phi(z)}(\phi(z))=\left(K_{z} \circ \phi^{-1}\right)(\phi z)\right)=K_{z}(z)
$$

is another consequence of (11). It proves (13), because

$$
K_{z}(z)=\left[K_{z}, K_{z}\right]>0 \text {. }
$$

Let us now prove the following proposition: 
Proposition 3.4. For each $z \in \partial_{0} \omega$, the space $N^{j} H_{k}$ contains a unique $f$ such that $f(z)=1$ and $f=f \circ \Psi$ for every $\Psi \in G$ that fixes $z$.

Proof: It follows from (12) and (13) that $f=\frac{K_{z}}{K_{z}(z)}$ satisfies the required properties. To prove the uniqueness, denote by $e_{1}$ the point $(1,0, \ldots, 0)$ and assume $z=e_{1}$ without loss of generality. The invariance $f=f \circ \Psi$ for every $\Psi \in S O(n)$ that fixes $e_{1}$ shows then, for $f=N^{j} p$, $p \in H_{k}$ and for each $w_{1}$, that $\left(w_{2}, \ldots, w_{n}\right) \mapsto p\left(w_{1}, w_{2}, \ldots, w_{n}\right)$ is a polynomial in $w_{2}^{2}+\cdots+w_{n}^{2}$. Since $p$ is a homogeneous holomorphic polynomial of degree $k$ on $\mathbb{C}^{n}$ that satisfies $p\left(e_{1}\right)=1, p$ therefore has the form

$$
p(w)=\sum_{i=1}^{r} c_{i}\left(w_{2}^{2}+\cdots+w_{n}^{2}\right)^{i} w_{1}^{k-2 i}
$$

where $r=\left[\frac{k}{2}\right]$ and $c_{0}, \ldots, c_{n}$ are constants, $c_{0}=1$. Differentiation of (14) gives

$$
\square p(w)=\sum_{i=0}^{r-1} b_{i}\left(w_{2}^{2}+\cdots+w_{n}^{2}\right)^{i} w_{1}^{k-2 i-2}
$$

where

$$
b_{i}=(k-2 i)(k-2 i-1) c_{i}+(2 i+1)(n+2 i) c_{i+1},
$$

for all $i=0, \ldots, r-1$. Since $\square p=0$, (15) vanishes, so that $b_{i}=0$ for all $i$ and (16) successively determines $c_{1}, \ldots, c_{r}$. This proves the proposition.

Our next result is the following:

Theorem 3.5. The irreductible representations $S_{j_{1} k_{1}}$ and $S_{j_{2} k_{2}}$ of $G$ in $N^{j_{1}} H_{k_{1}}$ and $N^{j_{2}} H_{k_{2}}$ respectively are inequivalent when $\left(j_{1}, k_{1}\right) \neq$ $\left(j_{2}, k_{2}\right)$. More precisely, suppose $T: N^{j_{1}} H_{k_{1}} \rightarrow N^{j_{2}} H_{k_{2}}$ is linear and commutes with $G$. Then

1. $T=0$ when $\left(j_{1}, k_{1}\right) \neq\left(j_{2}, k_{2}\right)$,

2. when $\left(j_{1}, k_{1}\right)=\left(j_{2}, k_{2}\right)$, there is a constant $c$ such that $T=c I$ where $I$ is the identity operator.

Proof: The assertion 2 follows from Schur's Lemma, as well as the following assertion: either $T=0$ or $T$ is invertible when $\left(j_{1}, k_{1}\right) \neq$ $\left(j_{2}, k_{2}\right)$. Let us prove that $T=0$ in the latter case. 
Let $K_{z}^{1} \in N^{j_{1}} H_{k_{1}}$ and $K_{z}^{2} \in N^{j_{2}} H_{k_{2}}$ be as in Theorem 3.3. If $\Psi \in G$ fixes $z$, then

$$
\left(T K_{z}^{1}\right) \circ \Psi=T\left(K_{z}^{1} \circ \Psi\right)=T K_{z}^{1}
$$

by (12), since $T$ commutes with $G$. By Proposition $3.4,(17)$ shows that to every $z \in \partial_{0} \omega$ corresponds a constant $c(z)$ such that $T K_{z}^{1}=c(z) K_{z}^{2}$. Hence $T K_{z}^{1}(z)=c(z) K_{z}^{2}(z)$. By (13), $K_{z}^{2}(z)$ is independent of $z$. If $w=$ $\phi(z), \phi \in G$, then by $(11),\left(T K_{w}^{1}\right)(w)=T\left(K_{z}^{1} \circ \phi^{-1}\right)(\phi(z))=T K_{z}^{1}(z)$.

We conclude from this that $c(z)=c$, the same for all $z \in \partial_{0} \omega$.

If $f \in N^{j_{1}} H_{k_{1}}$, (10) shows that

$$
f=\int_{\partial_{0} \omega} f(\zeta) K_{\zeta}^{1} d \sigma(\zeta)
$$

Apply $T$ to (18) and use $T K_{\zeta}^{1}=c K_{\zeta}^{2}$ :

$T f=c \int_{\partial_{0} \omega} f(\zeta) K_{\zeta}^{2} d \sigma(\zeta)=c \pi_{j_{2} k_{2}} f$, for every $f \in N^{j_{1}} H_{k_{1}}$. If $\left(j_{1}, k_{1}\right) \neq\left(j_{2}, k_{2}\right), \pi_{j_{1} k_{1}} f=0$ and hence $T f=0$. This proves the theorem.

\section{4. $G$-invariant spaces on $\partial_{0} \omega$}

For $\Omega \subset Q$ the algebraic sum of all $N^{j} H_{k}$ with $(j, k) \in \Omega$ will be denoted by $E_{\Omega}$. We adopt the convention that $E_{\Omega}=\{0\}$ when $\Omega$ is empty.

The letter $X$ will stand for any of the Banach spaces $\mathcal{C}\left(\partial_{0} \omega\right)$ or $L^{p}\left(\partial_{0} \omega, d \sigma\right), 1 \leq p<\infty$. The $X$-closure of $E_{\Omega}$ will be denoted by $X_{\Omega}$. Trivially, every $X_{\Omega}$ is a $G$-invariant closed subspace in $X$. The proof of the converse is the main topic of the section. In the sequel, we write $\mathcal{C}$ for $\mathcal{C}\left(\partial_{0} \omega\right)$ and $L^{p}$ for $L^{p}\left(\partial_{0} \omega, d \sigma\right)$.

We begin with the case $X=L^{2}$ :

Theorem 4.1. If $Y$ is a $G$-invariant closed subspace of $L^{2}\left(\partial_{0} \omega, d \sigma\right)$ and if $\Omega$ is the set of all $(j, k) \in Q$ such that $\pi_{j k} Y \neq\{0\}$, then $Y=$ $\left(L^{2}\right)_{\Omega}$.

Proof: Pick $(j, k) \in \Omega$. Since $Y$ is $G$-invariant and $\pi_{j k}$ commutes with $G, \pi_{j k} Y$ is a nontrivial invariant subspace of $N^{j} H_{k}$. By Theorem 3.1, we have therefore $\pi_{j k} Y=N^{j} H_{k}$.

Let $Y_{0}=\left\{f \in Y: \pi_{j k} f=0\right\}$, and let $Y_{1}$ be the orthogonal complement of $Y_{0}$ in $Y$. Then $Y_{0}$ is $G$-invariant, hence so is $Y_{1}$. Moreover, $\pi_{j k}: Y_{1} \rightarrow$ $N^{j} H_{k}$ is an isomorphism whose inverse we denote by $\Lambda$. 
Choose $(r, s) \in Q,(r, s) \neq(j, k)$, and consider the linear map $T=$ $\pi_{r s} \circ \Lambda: N^{j} H_{k} \rightarrow N^{r} H_{s}$. It is clear that $T$ commutes with $G$; hence, by Theorem 3.5, $T=0$ and $\pi_{r s} Y_{1}=\{0\}$ for every $(r, s) \neq(j, k)$. By Corollary 2.4, $Y_{1}$ is a nontrivial subspace of $N^{j} H_{k}$, isomorphic to $N^{j} H_{k}$; since $Y_{1}$ is $G$-invariant, then $Y_{1}=N^{j} H_{k}$ by Theorem 3.1. Furthermore, $N^{j} H_{k} \subset Y$, for every $(j, k) \in \Omega$; in other words, $\left(L^{2}\right)_{\Omega} \subset Y$. Since $\pi_{r s} Y=\{0\}$ for every $(r, s) \neq(j, k)$, a second look at Corollary 2.4 completes the proof of the theorem.

We shall need the following three lemmas:

Lemma 4.2. If $f \in X$, then $\phi \rightarrow f \circ \phi$ is a continuous map of $G$ into $X$.

Proof: If $\varepsilon>0$, then $\|f-g\|<\varepsilon$ for some $g \in \mathcal{C}$. There is a neighbourhood $N$ of the identity in $G$ such that $\|g-g \circ \phi\|_{\infty}<\varepsilon$ for every $\phi \in N$. Since $|f-f \circ \phi| \leq|f-g|+|g-g \circ \phi|+|(g-f) \circ \phi|$, we obtain $\|f-f \circ \phi\|<3 \varepsilon$ for every $\phi \in N$.

Lemma 4.3. If $Y$ is a $G$-invariant closed subspace of $X$, then $Y \cap \mathcal{C}$ is dense in $Y$.

Proof: Pick $f \in Y$, choose $N$ as in the proof of Lemma 4.2, let $\Lambda$ : $G \rightarrow[0, \infty)$ be continuous, with support in $N$ and $\int_{G} \Lambda d \phi=1$.

Define

$$
g=\int_{G} \Lambda(\phi) f \circ \phi d \sigma
$$

The integrand is a continuous $Y$-valued function. Hence $g \in Y$. If $\Psi \in G$ is such that $\Psi\left(e_{1}\right)=\zeta$, where $e_{1}=(1,0, \ldots, 0)$, the invariance of the Haar measure shows that

$$
g(\zeta)=\int_{G} \Lambda\left(\phi \psi^{-1}\right) f\left(\phi\left(e_{1}\right)\right) d \sigma
$$

Thus, $g \in Y \cap \mathcal{C}$. Finally, the relation

$$
f-g=\int_{N} \Lambda(\phi)(f-f \circ \phi) d \phi
$$

proves that $\|f-g\|<3 \varepsilon$, since $\|f-f \circ \phi\|<3 \varepsilon$ if $\phi \in N$.

Lemma 4.4. If $Y \subset \mathcal{C}, Y$ is a $G$-invariant space, and some $g \in \mathcal{C}$ is not in the uniform closure of $Y$, then $g$ is not in the $L^{2}$-closure of $Y$.

Proof: There is a regular complex Borel measure $m$ on $\partial_{0} \omega$ such that $\int f d m=0$ for all $f \in Y$, but $\int g d m=1$. There is a neighbourhood 
$N$ of the identity in $G$ such that $\operatorname{Re} \int g \circ \phi d m>\frac{1}{2}$ for every $\phi \in N$. Associate $\Lambda$ to $N$ as in the proof of Lemma 4.3, and define $L \in \mathcal{C}^{*}$ by

$$
L(h)=\int_{\partial_{0} \omega} d m(\zeta) \int_{G} \Lambda(\phi) h(\phi(\zeta)) d \phi .
$$

The Schwarz inequality shows that the square of the absolute value of the inner integral in (19) is at most

$$
\left(\int_{G}|\Lambda(\phi)|^{2} d \phi\right)\left(\int_{G}|h(\phi(\zeta))|^{2} d \phi\right)=c\|\Lambda\|_{2}^{2} \int_{\partial_{0} \omega}|h|^{2} d \sigma
$$

so that

$$
|L(h)| \leq c\|m\|\|\Lambda\|_{2}\|h\|_{2} .
$$

Thus $L$ extends to a bounded linear functional on $L^{2}$. If we interchange the two integrals in (19), we see that $L(h)=0$ for every $h \in Y$, whereas $\operatorname{Re} L(g) \geq \frac{1}{2}$. This completes the proof.

We can now prove the following theorem, announced at the beginning of the section:

Theorem 4.5. If $Y$ is a $G$-invariant closed subspace of $X$, and if $\Omega$ is the set of all $(j, k) \in Q$ for which $\pi_{j k} Y \neq\{0\}$, then $Y=X_{\Omega}$.

Proof: Observe first that the domain of $\pi_{j k}$ can be extended to $L^{1}$, by (10). Define $\tilde{Y}$ to be the $L^{2}$-closure of $Y \cap \mathcal{C}$. Since $Y$ is $X$-closed, $Y \cap \mathcal{C}$ is uniformly closed, so that Lemma 4.4 gives

$$
\tilde{Y} \cap \mathcal{C}=Y \cap \mathcal{C} .
$$

Observe next that $Y \cap \mathcal{C}$ is $L^{2}$-dense in $\tilde{Y}$, by definition, and is $X$-dense in $Y$, by Lemma 4.3. Since $\int_{\partial_{0} \omega}\left|K_{\zeta}(z)\right|^{2} d \sigma(\zeta)=K_{z}(z)=c$ is independent of $z$ by (13), we get $\left|K_{\zeta}(z)\right|=\left|\int_{\partial_{0} \omega} K_{\zeta}(w) \overline{K_{z}(w)} d \sigma(w)\right| \leq c$ for all $\zeta, z$ in $\partial_{0} \omega$, so that by $(10)$, we obtain

$$
\left|\pi_{j k} f(z)\right| \leq c\|f\|_{L^{p}}
$$

for all $p \in[1, \infty]$ and $z \in \partial_{0} \omega$. Hence each $\pi_{j k}$ is $X$-continuous as well as $L^{2}$-continuous. It follows that $\pi_{j k} Y=\{0\}$ if and only if $\pi_{j k} \tilde{Y}=\{0\}$. Theorem 4.1 shows therefore that $\tilde{Y}$ is the $L^{2}$-closure of $E_{\Omega}$. Since $E_{\Omega} \subset \mathcal{C}$, another application of Lemma 4.4 gives

$$
\tilde{Y} \cap \mathcal{C}=\text { uniform closure of } E_{\Omega} .
$$

Since $Y \cap \mathcal{C}$ is $X$-dense in $Y$ (Lemma 4.3), (20) and (21) imply that $Y$ is the $X$-closure of $E_{\Omega}$. This is the assertion of the theorem.

We deduce the following corollary: 
Corollary 4.6. Suppose $T: X_{1} \rightarrow X_{2}$ is a continuous linear map that commutes with $G$. Then there exist $c(j, k) \in \mathbb{C}$, for every $(j, k) \in Q$, such that

$$
T f=c(j, k) f \text { for all } f \in N^{j} H_{k} .
$$

The numbers $c(j, k)$ determine $T$.

Proof: The map $\pi_{r s} T: N^{j} H_{k} \rightarrow N^{r} H_{s}$ commutes with $G$, hence by Theorem 3.5, $\pi_{r s} T f=0$ if $f \in N^{j} H_{k}$ and $(r, s) \neq(j, k)$. Consequently, $T\left(N^{j} H_{k}\right)$ is a (finite dimensional, hence closed) $G$-invariant subspace of $X_{2}$ that is annihilated by $\pi_{r s}$ wherever $(r, s) \neq(j, k)$. By Theorem 4.5, $T\left(N^{j} H_{k}\right)=N^{j} H_{k}$ or $\{0\}$. Now (22) follows from Theorem 3.5.

\section{Proof of Theorem 1.1}

Assume, to reach a contradiction, that for some $p \in[1, \infty), p \neq 2$, there is a continuous linear projection $P$ of $L^{p}$ onto $H^{p}=H^{p}(\omega)$. Define $T: L^{p} \rightarrow H^{p}$ by

$$
T f=\int_{G}\left[P\left(f \circ \phi^{-1}\right)\right] \circ \phi d \phi .
$$

It is proved in [R2] that $T$ is then a continuous linear projection of $L^{p}$ onto $H^{p}$ that commutes with $G$. Thus $T$ is as in (22), (the case where $X_{1}=X_{2}=L^{p}$ in Corollary 4.6), with $c(j, k)=1$ for all $(j, k) \in \mathbb{Z}_{+} \times \mathbb{Z}_{+}$ and $c(j, k)=0$ whenever $j<0$ and $k \geq 0$. Since it is well known (cf. e.g. $[\mathbf{H}])$ that

$$
H^{2}=\sum_{(j, k) \in \mathbb{Z}_{+} \times \mathbb{Z}_{+}} \oplus N^{j} H_{k},
$$

this implies that $T f$ is the boundary value function of the Szegö projection of $f$. We conclude that the Szegö projection of $\omega$ is $L^{p}$-bounded for some $p \in[1, \infty), p \neq 2$. This contradicts Theorem 1.2.

\section{References}

[Be Bo] D. BeKollé AND A. Bonami, Estimates for the Bergman and Szegö projections in two symmetric domains of $\mathbb{C}^{n}$, Prépublications Math. Orsay (1993).

[BCK] C. A. Berger, L. A. Coburn and A. Koranyi, Opérateurs de Wiener-Hopf sur les sphères de Lie, C. R. Aca. Sc. Paris 290 (1980), 989-991. 
[Co We] R. R. Colfman and G. Weiss, "Analyse harmonique non commutative sur certains espaces homogènes," Lecture Notes in Math. 242, Springer, New York, 1971.

[H] L. K. HUA, "Harmonic Analysis of functions of several complex variables in the classical domains," Transl. Math. Monographs 6, Amer. Math. Soc., Providence, 1963.

[K] A. KoranyI, Complex Analysis and symmetric domains, Ecole CIMPA, Université de Poitiers (1988).

[KV] A. Koranyi and S. VAGI, Singular integrals on homogeneous spaces and some problems of classical analysis, Ann. Scuola Norm. Sup. Pisa 25 (1971), 575-648.

[R1] W. Rudin, "Function Theory in the unit ball of $\mathbb{C}^{n}$," Springer-Verlag, 1980.

[R2] W. Rudin, Projections on invariant subspaces, Proc. Amer. Math. Soc. 13 (1962), 429-432.

[V] S. VAGI, Harmonic analysis in Cartan and Siegel domains, $M A$ A Studies in Mathematics 13, Studies in Harmonic Analysis (J. M. Ash, ed.) (1976).

[W] P. WojtaszczyK, Hardy spaces on the complex ball are isomorphic to Hardy spaces on the disc, $1 \leq p<\infty$, Ann. of Math. (2)118 (1983), 21-34.

[Wo] T. M. Wolniewicz, Independent inner functions in the classical domains, Glasgow Math. J. 29 (1987), 229-236.

\author{
Department of Mathematics \\ Faculty of Science \\ P.O. Box 812 \\ University of Yaoundé \\ Yaoundé \\ CAMEROON
}

Rebut el 18 de Juny de 1993 